\title{
CONSENTIMIENTO INFORMADO. PERCEPCIÓN DE PACIENTES. REVISIÓN DOCUMENTAL 1995-2013*
}

\author{
Título corto: Consentimiento informado-percepción pacientes \\ MARÍA TERESA ESCOBAR LÓPEZ ${ }^{1}$ \\ ${ }^{1}$ Doctora en Bioética, Universidad Militar Nueva Granada, Bogotá D.C., Colombia. \\ Correspondencia: María Teresa Escobar López, carrera 58 b No. 127d-37, \\ cel.3014307902.maria.escobar@unimilitar.edu.co
}

Recibido: 16 de octubre de 2016 Aceptado: 27 de junio de 2017

\section{Resumen}

Objetivo. Revisar literatura descriptiva y analítica sobre consentimiento informado (CI), publicada entre 1995 y 2013, centrando el interés en los estudios que muestran la percepción de los pacientes en atención clínica.

Métodos. El proceso de indagación consistió en la revisión de literatura descriptiva y analítica sobre consentimiento informado, en formatos digitales y en físico, centrando el interés en los estudios que muestran la percepción de los pacientes en atención clínica. Se compiló un total de 78 documentos entre artículos científicos, trabajos de grado, libros y capítulos de libro, publicados entre los años 1995 y 2013. Con estos aspectos de contenido se elaboró una hoja de cálculo (matriz en Excel), con resumen, consideraciones teóricas, metodológicas, observaciones y conclusiones.

Resultados. Del total de documentos consultados, solamente dos estudios particularizan sobre un área de atención clínica: la oncología, y apenas algunos tratan la percepción del $\mathrm{CI}$ en atención clínica de pacientes. Los demás, son de teoría sobre $\mathrm{CI}, \mathrm{CI}$ e investigación o se refieren al él en general y versan sobre distintos temas, teniendo en cuenta la percepción de profesionales y expertos. Finalmente, en ningún documento se mencionan posturas teóricas sobre percepción y todos los documentos refieren problemas frente a su aplicación e implementación. En todos los casos, la autonomía se entiende como una expresión de voluntad que requiere ser aplicada, lo cual es determinante para el CI.

Conclusiones. Existen pocos estudios de percepción en pacientes y en ninguno se explica qué se entiende por este concepto. Por el contrario, es generalizado en los escritos revisados, lo que evidencia problemas relacionados con la aplicación e implementación del CI.

Palabras clave: consentimiento informado; percepción; revisión documental.

\section{INFORMED CONSENT. PATIENTS' PERCEPTION. DOCUMENTS REVIEW, 1995-2013}

\section{Summary}

Objective: To review descriptive and analytical literature on informed consent (IC), published between 1995 and 2013; focusing on studies that show the patients' perception of clinical care. 
Methods: The investigation process consisted of reviewing descriptive and analytical literature related to informed consent, in both digital and physical formats, while focusing on studies that show the patients' perception of clinical care. A total of 78 documents were compiled, including scientific articles, university theses, complete books, and book chapters, all published between 1995 and 2013. Taking all these into account, a spreadsheet (Excel matrix) was elaborated, including a summary, theoretical considerations, methodologies, observations and conclusions.

Results: Of the total number of documents consulted, only two studies specify a clinical care area: oncology, and just a few deal with the perception of IC in clinical patient care. The others are theoretical in their approach to IC, IC and research, or they refer to it in general terms and deal with different topics, taking into account the perception of professionals and experts. Finally, theoretical positions on perceptions are not mentioned in any of the documents, and all the documents refer to problems regarding its application and implementation. In all cases, autonomy is understood as an expression of will that needs to be applied, which is decisive for IC.

Conclusions: There are only a few studies of perception in patients and none of them explain what is understood by this concept. On the contrary, IC is generalized in the reviewed writings, which shows problems related to the application and implementation of IC.

Keywords: informed consent; perception; documentary review.

\section{CONSENTIMENTO INFORMADO. PERCEPÇÃO DE PACIENTES. REVISÃO DOCUMENTAL 1995-2013}

\section{Resumo}

Objetivo. Revisar literatura descritiva e analítica sobre consentimento informado (CI), publicada entre 1995 e 2013, centrando o interesse nos estudos que mostram a percepção dos pacientes em atenção clínica.

Métodos. O processo de indagação consistiu na revisão de literatura descritiva e analítica sobre consentimento informado, em formatos digitais e em físico, centrando o interesse nos estudos que mostram a percepção dos pacientes em atenção clínica. Foi compilado um total de 78 documentos entre artigos científicos, trabalhos de conclusão de curso, livros e capítulos de libro, publicados entre os anos 1995 e 2013. Com estes aspectos de conteúdo foi elaborada uma folha de cálculo (matriz em Excel), com resumo, considerações teóricas, metodológicas, observações e conclusões.

Resultados. Do total de documentos consultados, somente dois estudos particularizam sobre uma área de atenção clínica: a oncologia, e apenas alguns tratam a percepção do $\mathrm{CI}$ em atenção clínica de pacientes. Os demais são de teoria sobre $\mathrm{CI}, \mathrm{CI}$ e pesquisa ou se referem ao tema em geral e versam sobre diferentes temas, levando em conta a percepção de profissionais e expertos. Finalmente, em nenhum documento se mencionam posturas teóricas sobre percepção e todos os documentos referem problemas diante da sua aplicação e implementação. Em todos os casos, a autonomia se entende como uma expressão de vontade que requer ser aplicada, o que é determinante para o CI.

Conclusões. Existem poucos estudos de percepção em pacientes e em nenhum se explica que se entende por este conceito. Pelo contrário, é generalizado nos escritos revisados, o que evidencia problemas relacionados com a aplicação e implementação do CI.

Palavras-chave: consentimento informado; percepção; revisão documental. 


\section{Introducción}

Un tema central para la bioética es el consentimiento informado $(\mathrm{CI})$, enunciado generalmente como expresión de autonomía individual en la práctica clínica y de investigación. Los problemas que se presentan en su aplicación e implementación en el área de la salud han ido desbordando los intentos de dar respuestas, principalmente desde posturas legales y técnico-instrumentales. Un hecho es fácilmente comprobable: el problema se ha abordado en la mayoría de los casos sin tener en cuenta la visión de los pacientes sobre ese instrumento.

El CI se ha implementado en la práctica clínica y la investigación desde que el concepto se enunció en el Código de Núremberg, en 1948, y para la investigación en 1957 cuando, desde la jurisprudencia, se incorporó a la clínica humana. El Caso Salgo contra Stanford Jr. University (Civ. No. 17045. Primer Dist., Div. Uno. Oct 22, 1957. Tribunal Supremo de California, en EE. UU.), es el primer caso documentado del uso de este concepto. Desde entonces, ha logrado un vertiginoso proceso de aceptación y ha permeado todos los escenarios del área de la salud. En unos casos lo ha hecho de forma sistemática, mientras que en otros ha enfrentado grandes dificultades, por lo que ha aparecido tardíamente y condicionado, en la mayoría de los escenarios, por exigencias legales. Un caso al respecto se presenta el Estudio histórico interpretativo del consentimiento informado en la odontología en Cundinamarca 1990-2010, desarrollado por la Dra. María Teresa Escobar López. Se trata de una investigación realizada como tesis de grado y que mereció la distinción Magna cum Laude, en la que su autora logró establecer que el proceso se implementó fundamentalmente por las exigencias de la secretaria de salud de Bogotá y que su contenido y forma se han ido estructurando solo a partir de la jurisprudencia.

En ciertas áreas, su inclusión no supera las tres décadas $y$, desde los postulados teóricos, se habla del CI en la toma de decisiones, como expresión del principio de autonomía, cuando se informa en forma oportuna, adecuada y veraz de los procedimientos a los cuales se someterá un individuo, ya sea en investigación o en atención en salud. En este sentido, acerca de las generalidades teóricas y consentimiento informado, existen tres trabajos excepcionales, desarrollados por Simón Lorda, Guy Durand, y Diego Gracia, quienes dan un tratamiento riguroso al tema: Teoría y práctica del Consentimiento Informado, Introducción Genérale a la Bioetiquey Ética Clínica (tomo II), respectivamente.
Esta revisión documental visibiliza el tratamiento que se ha dado al tema desde la investigación y la literatura científica. Sirve al propósito de delimitar objetivos específicos y recoger información sobre aspectos concretos del diseño, estrategias, pautas de seguimiento, criterios de selección y determinación del tamaño de muestra, entre otros, de investigaciones sobre percepción de los pacientes en diferentes áreas de atención en Bogotá y, sobre todo, para mostrar el panorama de lo publicado al respecto entre 1995 y 2013. ${ }^{1}$

\section{Aspectos generales}

Con la inclusión del CI en la actividad clínica, en parte resultado de la exigencia social de respeto a la autonomía de los pacientes y plasmado en la jurisprudencia que le proporcionó el respaldo legal en un primer momento, se inició un nuevo proceso en la atención clínica, en el cual el paciente empezó a exigir ser informado y escuchado en las decisiones relacionadas con su salud. Como ya se anticipó, este nuevo procedimiento inició en la década de 1960, en EE. UU., y fue fortaleciéndose con el transcurrir del tiempo hasta convertirse en un paso ineludible en la relación profesional-paciente en salud (2). En Colombia, el uso del CI se inicia en la atención médica hospitalaria, desde donde se extiende en un comienzo a otras áreas y especialidades médicas no hospitalarias y, en las dos últimas décadas, involucra más fuertemente otras profesiones como la enfermería y la odontología. $\mathrm{Al}$ igual que en la clínica humana norteamericana, aparece fundamentalmente como una exigencia legal y básicamente como documento escrito.

Es importante resaltar la dinámica social de la que parte la implementación de este instrumento: sociedades en las que la autonomía individual es más tenida en cuenta, donde se pide mayor respeto por los derechos individuales. Es decir, sociedades donde las exigencias de los pacientes de participar en las decisiones que tienen que ver con su salud, su cuerpo y su vida, se han instaurado desde un punto de vista legal. De esta manera, se ha ido incluyendo el consentimiento informado en leyes, resoluciones, protocolos de manejo, exigencias de los entes reguladores, de calidad y de control, entre otros (2)

\footnotetext{
1 Tanto en investigación cualitativa como cuantitativa y en clínica epidemiológica se resalta la valiosa revisión documental y el estado de la cuestión existentes. Ver especialmente: Argimon, J. y Jimenez J. Métodos de investigación clínica y epidemiológica (2004, pp. 117- 124); Castellanos C. Estrategias de investigación en metodología cualitativa (1996) y Passeron, JC., El razonamiento sociológico (2011).
} 
A pesar del supuesto teórico del que parte, la implementación y el análisis de cómo se desarrolla y aplica en los diferentes ámbitos clínicos (2) no se realiza de la manera más adecuada. Se suele hablar del CI en general, bajo los mismos parámetros en todas las dimensiones de atención en salud, clínica hospitalaria, ambulatoria, en cuidados de pacientes crónicos, agudos, atención primaria, etc., sin tener en cuenta, por ejemplo, diferentes contextos sociales y culturales, los cuales también están influenciados por la forma como se presta la atención en salud, a partir de las leyes que la regulan.

Además, en este complejo panorama, la percepción de los pacientes se ha estudiado poco y, en general, el proceso en sí ha pasado desapercibido. Aspectos que son de gran relevancia para el paciente, tales como: el nivel de comprensión de documentos escritos, el lenguaje utilizado, las circunstancias en que se da y solicita la atención, así como las diferencias culturales y educacionales, no se suelen tener en cuenta. Estos son algunos de los puntos más problemáticos, pero existen más. ${ }^{2}$ Por ejemplo, cuando se considera que, constitucionalmente, Colombia es una sociedad multicultural, pluriétnica; un país donde subsisten grandes diferencias e inequidades sociales, y de todo tipo, queda claro que existen grandes barreras que impiden una adecuada y activa participación entre el paciente y el profesional de salud. Es decir, la comunicación está fuertemente condicionada, situación que debe ser analizada a profundidad.

\section{Consideraciones necesarias con respecto a la percepción en pacientes}

La percepción (recibir imágenes, impresiones o sensaciones externas, a partir de los sentidos, comprender o conocer algo) constituye una herramienta fundamental en la práctica investigativa de carácter cualitativo. Este concepto empieza a ser ampliamente utilizado desde el siglo XIX y ha facilitado la resolución de problemas que la investigación cuantitativa no lograba resolver.

A partir de la definición misma de percepción se desprende una primera dificultad: la identificación frecuente de percibir y conocer, es decir, que son analizados como sinónimos. Sin embargo, para expertos en el tema como Luz Vargas (3), esta identificación representa una dificul-

2 Al respecto, resulta interesante el trabajo de Pablo Simon Lorda, Problemas prácticos sobre consentimiento informado, elaborado a partir de un coloquio de 2002 que reunió expertos en el tema y que visibiliza ampliamente la problemática. tad para su aplicación como herramienta de observación y estudio de algunos fenómenos. Por eso, es importante aclarar que percibir, si bien hace parte del conocer, no se puede utilizar como equivalente de conocer en investigación cualitativa, pues el segundo deja por fuera aspectos fundamentales relacionados con el contexto donde se desarrolla la experiencia sensible.

Por otro lado, en psicología, donde el término es ampliamente utilizado, se suele hablar de la percepción como una función psíquica que, por medio de los sentidos, permite a la persona captar estímulos para procesar, posteriormente, la información que le llega del exterior, la cual puede ser sensorial o cognitiva.

En bioética, por su parte, resulta importante esta visión de Luz Vargas, para quien la percepción es una forma de conducta que comprende el proceso de selección y elaboración simbólica de la experiencia sensible, que tiene como límites las capacidades biológicas humanas y el desarrollo de la cualidad innata del ser humano para la producción de símbolos a través de lo que experimenta (3).

Ahora bien, desde la óptica cualitativa, la percepción, tiene particularidades interesantes al incluir los objetos o circunstancias del entorno e implica la forma como se elaboran sistemas culturales e ideológicos específicos, construidos y reconstruidos por el grupo social (en este caso los pacientes que asisten a los servicios de atención clínica y hospitalaria en salud y los que se someterse a tratamientos en los diferentes servicios de las instituciones de atención). La percepción, entendida en un contexto histórico social, implica pues una ubicación temporal y espacial particular. De esta manera, se comprende que las vivencias de los pacientes están influidas por circunstancias cambiantes. La pregunta fundamental es entonces si esto se tiene en cuenta en la relación profesionalpaciente, cuando de consentimiento informado se trata. Experiencias, actitudes, percepciones, están intimamente interrelacionados y son una herramienta fundamental para conocer cómo se incluyen en la atención clínica, en el proceso de consentimiento informado.

\section{Materiales y métodos}

Se adelantó una revisión documental descriptiva y analítica, centrando el interés en los estudios que muestran la percepción de los pacientes sobre la atención clínica. El proceso de indagación tuvo en cuenta materiales impresos y formatos digitales. El análisis de materiales digitales 
estuvo enfocado en la búsqueda de publicaciones indexadas, relacionadas con el tema. Para ello, se realizaron búsquedas en las siguientes bases de datos: Academic Search Complete; Biblioteca Virtual de Salud; Directory of Open Acces- Journals; Ebrary; Embase; Emerald; Global health; Jorunal Storage; Medine Ovid- Journals; Pubmed; REDALYC; Scientific Electronic ibrary Online; Science Direct; y Springer. El acceso a estas bases de datos se hizo por medio de los buscadores de la Universidad Nacional de Colombia, la Universidad El Bosque y la Universidad Militar Nueva Granada. En cuanto al material físico, se hicieron búsquedas de publicaciones académicas en las bibliotecas de las tres universidades mencionadas.

Para una indagación digital más exhaustiva, se filtró la subárea bioética, utilizando como términos de búsqueda: consentimiento + informado, percepción + pacientes y consentimiento + autonomía + pacientes. Se compiló un total de 78 documentos entre artículos científicos, trabajos de grado, libros y capítulos de libro, publicados entre 1995 y 2013 y, a partir de ello, se elaboró una matriz con los siguientes aspectos de contenido: 1) resumen, 2) consideraciones teóricas y metodológicas, 3) observaciones y 4) conclusiones. Los documentos se dividieron en cuatro grupos, de acuerdo con su contenido en: documentos de investigación, de teoría sobre el CI en general, percepción en profesionales y percepción en pacientes. Finalmente, se realizaron gráficas sobre distribución por áreas y años, para facilitar la interpretación, pues la investigación es eminentemente cualitativa.

\section{Resultados}

De la lectura de los materiales, se concluye que mayoría de los 78 documentos consultados se detiene en el tema de manera general, analizando diversos aspectos del CI, tales como si se incluyen las condiciones del proceso de consentimiento; la información, la capacidad y la voluntariedad; sus funciones, limites y excepciones de su uso. Pero, para el análisis presente, luego de una revisión fueron agrupados así: a) 30 de teoría del CI y percepción en profesionales (algunos hacen énfasis en conocimiento y recomendaciones para profesionales y estudiantes), además, en ellos se indaga a profesionales de diferentes áreas, más en investigación que en la práctica clínica; b) 21 documentos de $\mathrm{CI}$ en investigación; c) uno sobre el documento escrito exclusivamente y d) 26 de percepción sobre CI en pacientes, la mayoría de estos estudios en investigación (tabla 1).
Las investigaciones, en general, presentan preocupaciones por las dificultades en el proceso del CI y muchas de ellas están centradas en los documentos escritos. Asimismo, se indaga sobre la forma de comprender y cumplir con el CI en distintos países, teniendo en cuenta la normativa local y, en algunos casos, se hace intervención en los grupos para mejorar el proceso de aplicación, cuando se utilizan metodologías, como por ejemplo investigación-acción-participativa (IAP), partiendo de asegurar que existen problemas en el CI. Estudios directamente enfocados en la percepción del CI en pacientes, son escasos. Pero en ninguno de los estudios se incluye postura teórica con respecto al concepto mismo de percepción.

Con el paso del tiempo, el número de publicaciones sobre el tema se ha incrementado, sobre todo en los últimos cinco años. Además, es marcado el énfasis en el contexto de la investigación o en pacientes de medicina crítica, como oncología, cirugía, etc. En general, en los documentos se habla del respeto por la autonomía o de la importancia de la autonomía como principio.

Por otra parte, en una elevada proporción de los escritos se menciona la idea de entender el CI como expresión de la voluntad del sujeto, la necesidad de implementarlo, la capacidad y la voluntad como elementos esenciales o también se afirma que no hay mayor novedad con respecto a los postulados teóricos y jurisprudencia que dieron cabida en la relación entre profesionales de salud y pacientes.

En los estudios se habla del CI de manera genérica, sin tener en cuenta la percepción del paciente. Todos refieren, de alguna manera, que existen problemas alrededor de su implementación y aplicación, corroborando lo expresado en estudios anteriores y en los pronunciamientos internacionales, los cuales sugieren especial cuidado al respecto: distintos escritos de la UNESCO, cuadernos de bioética de la OPS, y otras publicaciones de fundaciones como Grifolis i Lucas, donde se expresa esta preocupación.

El concepto de percepción no es trabajado en los documentos de manera teórica específica, lo que lleva a inferir que se incluye en la mayoría de los casos como un sinónimo o se equipara a una experiencia, sin un soporte para inquirir la postura de la que se parte. solo se hace referencia a la percepción, sin ahondar en ella. En ocasiones se utilizan métodos cuantitativos y revisiones desde la mirada clínica en salud, se refieren estadísticas 
Tabla 1. Resultados del análisis de las fuentes consultadas

\begin{tabular}{|c|c|c|}
\hline Autor & Año & Temática \\
\hline Benítez et al. (4) & 2002 & \multirow{38}{*}{$\begin{array}{l}\text { Teoría sobre el } \mathrm{Cl} \text {, métodos de implementación y } \\
\text { recomendaciones generales y específicas sobre } \mathrm{Cl} \text {, } \\
\text { cumplimiento de normativas y uso y diseño de formatos de } \\
\mathrm{Cl} \text {, pertinencia de uso de } \mathrm{Cl} \text {. }\end{array}$} \\
\hline Bettman y Lanckton (5) & 2012 & \\
\hline Cañete et al. (6) & 2012 & \\
\hline Cheatle y Savage (7) & 2012 & \\
\hline Clarke (8) & 2003 & \\
\hline Delany (9) & 2005 & \\
\hline Gómez y González (10) & 2012 & \\
\hline Gopal et al. (11) & 2012 & \\
\hline Lynoe et al (12) & 2001 & \\
\hline Mitchell (13) & 2003 & \\
\hline Padhy et al. (14) & 2011 & \\
\hline Piera y Ortega (15) & 2012 & \\
\hline Skillern et al. (16) & 2013 & \\
\hline Dávila y Pérez (17) & 2005 & \\
\hline De Siqueira (18) & 2009 & \\
\hline Berrocal (19) & 2004 & \\
\hline Cabrales et al. (20) & 2012 & \\
\hline Macías (21) & 2006 & \\
\hline Sankar (22) & 2004 & \\
\hline Kapp (23) & 2007 & \\
\hline Reyes, Águila, Llanes, Robaina y de Sanfiel (24) & 2007 & \\
\hline Aguilar (25) & 2008 & \\
\hline Figueroa (26) & 2012 & \\
\hline Siegal, Bonnie, Appelbaum (27) & 2012 & \\
\hline Stoljar (28) & 2011 & \\
\hline García (29) & 2007 & \\
\hline Wiesner y Monsalve (30) & 2009 & \\
\hline Galán (31) & 2001 & \\
\hline Castaño (32) & 1997 & \\
\hline Fajardo y Ruano (33) & 2009 & \\
\hline Gómez y Soto (34) & 2002 & \\
\hline Echavarría y Martínez (35) & 2002 & \\
\hline Luque y Villota (36) & 1998 & \\
\hline Parra (37) & 2002 & \\
\hline De Brigard (38) & 2004 & \\
\hline Devaninga (39) & 2012 & \\
\hline Herazo (40) & 2007 & \\
\hline Lanckton A van C (41) & 1996 & \\
\hline
\end{tabular}




\begin{tabular}{|l|l|l|}
\hline \multicolumn{1}{|c|}{ Autor } & Año & \multicolumn{1}{c|}{ Temática } \\
\hline Bismark et al.(42) & 2012 & $\begin{array}{l}\text { Percepción y problemas para los profesionales en } \\
\text { aplicación e implementación del consentimiento } \\
\text { informado. }\end{array}$ \\
\cline { 1 - 2 } Hipshman (43) & 1999 & \\
\cline { 1 - 2 } Ovalle (44) & 2009 & \\
\cline { 1 - 2 } Oppliger y Bascuñan (45) & 2011 & \\
\cline { 1 - 2 } Charry y Lopez (46) & 2005 & \\
\cline { 1 - 2 } Stoljar (47) & 2011 & \\
\hline
\end{tabular}

\begin{tabular}{|c|c|c|}
\hline Autor & Año & Temática \\
\hline Brown, Butow, Ellis, Boyle y Tattersall (48) & 2004 & \multirow{15}{*}{$\begin{array}{l}\text { Consentimiento informado en investigación, problemas, } \\
\text { aplicación, y comprensión de formatos. }\end{array}$} \\
\hline Cañete, et al. (49) & 2012 & \\
\hline Dawson y Kass (50) & 2005 & \\
\hline Ellenberg (51) & 1997 & \\
\hline Eyler, Mirzakhanian y Jeste (52) & 2005 & \\
\hline Gikonyo, Bejon, Marsh y Molyneux (53) & 2008 & \\
\hline Joffe, Cook, Cleary, Clark y Weeks (54) & 2001 & \\
\hline Pullman y Wang (55) & 2001 & \\
\hline Ordovás (56) & 2008 & \\
\hline Valle-Mansilla, Ruiz-Canela y Sulmasy (57) & 2008 & \\
\hline Ayuningtyas y Sondani (58) & 2010 & \\
\hline $\begin{array}{l}\text { Meneguin, Silmara, Zobolill, Domingues } \\
\text { y Nobrel (59) }\end{array}$ & 2011 & \\
\hline Garzón (60) & 2010 & \\
\hline Fernandes y Ardura (61) & 2012 & \\
\hline Geller (62) & 2012 & \\
\hline
\end{tabular}

\begin{tabular}{|l|l|l|}
\hline \multicolumn{1}{|c|}{ Autor } & Año & \multicolumn{1}{|c|}{ Temática } \\
\hline Clark et al. (63) & 2001 & \multirow{2}{*}{$\begin{array}{c}\text { Percepción de consentimiento informado en pacientes y/o } \\
\text { conocimiento sobre Cl consentimiento. }\end{array}$} \\
\cline { 1 - 2 } Enama et al. (64) & 2012 & \\
\cline { 1 - 2 } Fitzgerald et al. (65) & 2002 & \\
\cline { 1 - 2 } Hsiao-Lin H, LianHua H, y Fon-Jou H (66) & 2010 & \\
\cline { 1 - 2 } Kajja, Bimenya, y Smit (67) & 2011 & \\
\cline { 1 - 2 } Guix J. et al. (68) & 1999 & \\
\hline
\end{tabular}

\begin{tabular}{|l|l|l|}
\hline \multicolumn{1}{|c|}{ Autor } & Año & \multicolumn{1}{c|}{ Temática } \\
\hline Fenety, Harmana, Hoensb y Bassettd (69) & 2009 & $\begin{array}{l}\text { Estudio en profesionales sobre conocimientos, opiniones, } \\
\text { y prácticas acerca de la concepción sobre el Cl, en una } \\
\text { ocasión también en pacientes. }\end{array}$ \\
\cline { 1 - 2 } Gori et al. (70) & 2012 & \\
\cline { 1 - 2 } Hazen, Drotar, y Kodish (71) & 2007 & \\
\cline { 1 - 2 } Kušec et al. (72) & 2006 & \\
\cline { 1 - 2 } Leeper-Majors, Veale, Westbrook, y Reed (73) & 2003 \\
\cline { 1 - 2 } Gasull et al. (74) & 2002 \\
\cline { 1 - 2 } Nortes, Serra, y Tella (75) & 2011 & \\
\cline { 1 - 2 } Bello (76) & 2005 & \\
\cline { 1 - 2 } Jara y Bravo (77) & \\
\hline
\end{tabular}




\begin{tabular}{|l|l|l|}
\hline \multicolumn{1}{|c|}{ Autor } & Año & \multicolumn{1}{c|}{ Temática } \\
\hline Hung et al. (78) & 2011 & $\begin{array}{l}\text { Aprendizaje sobre el Cl con uso de multimedia u otras } \\
\text { ayudas audiovisuales. }\end{array}$ \\
\cline { 1 - 2 } Shin-Yuan H, Kuo-Liang H, y Wen-Ju Y (79) & 2011 & \\
\hline
\end{tabular}

\begin{tabular}{|l|c|c|}
\hline \multicolumn{1}{|c|}{ Autor } & Año & \multicolumn{1}{|c|}{ Temátical } \\
\hline Négrier et al. (80) & 1995 & $\begin{array}{l}\text { Efectos, eficacia y comprensión de la información médica; } \\
\text { toma de decisiones médicas. }\end{array}$ \\
\cline { 1 - 2 } $\begin{array}{l}\text { Nwomeh, Hayes, Caniano, Upperman, } \\
\text { y Kelleher (81) }\end{array}$ & 2009 & \\
\cline { 1 - 2 } $\begin{array}{l}\text { Olver, Whitford, Denson, Peterson, } \\
\text { y Olver (82) }\end{array}$ & 1995 & \\
\cline { 1 - 2 } Bespali, De Pena, Consens (83) & 2011 & \\
\cline { 1 - 2 } Cook, Damato, Marshall, y Salmon (84) & 2007 & \\
\cline { 1 - 2 } Hall, Div, y Morrison (85) & & \\
\hline
\end{tabular}

y se sacan conclusiones cualitativas a partir de datos estadísticos, pero se necesita sondear aspectos básicos e influyentes para el análisis completo, tales como el contexto en que se toman las decisiones, por ejemplo. En todos los casos, se parte de problemas en el CI en general como hecho comprobado

En otro aspecto, la queja más común suele ser la comprensión y comunicación deficientes o el conflicto con condicionantes en la atención. Se incluyen preocupaciones sustanciales tales como que mencionar efectos secundarios no es suficiente y que es importante mejorar la comunicación e implementar mecanismos de ayuda para la comprensión, aumentar el tiempo, entender que existen problemas relacionados con la industria de medicamentos, y también que la falta de tiempo en la atención es un limitante. Asimismo, se problematiza más alrededor de comprensión que de percepción, pero tampoco se muestra alguna postura teórica específica; algunos escritos hacen referencia a la confianza en la práctica clínica o en los profesionales y su equipo de trabajo: la confianza da para que el paciente crea que no debe tomar decisiones sino seguir las indicaciones sugeridas, afirman algunos de los encuestados.

Estudios realizados específicamente sobre comprensión del documento de $\mathrm{CI}$, tanto en clínica como en investigación, refieren poca comprensión de la información, pero enuncian que, aun así, se firma el documento. Se dan, con respecto a la forma como se obtiene el consentimiento, expresiones como la obtención del consentimiento ritual inflexible y estándar; muchos escritos están específicamente centrados en revisar aspectos legales y jurídicos del consentimiento informado. Un estudio en particular se remite al CI como soporte del respeto de los derechos humanos.

Finalmente, es importante resaltar que en muchos escritos las conclusiones están encaminadas a sugerir que el $\mathrm{CI}$ sea visto como proceso dinámico. Algunos alcanzan a identificar como problema el no particularizar el área de atención, pero sin llegar a establecer problemas de percepción de forma contundente.

La tabla 1 presenta un consolidado de las fuentes consultadas, agrupando a los autores de acuerdo con las temáticas expuestas en cada uno de sus textos.

\section{Discusión y conclusiones}

Desde la fundamentación de la bioética y la discusión actual sobre metodologías utilizadas, se ha venido insistiendo en la necesidad de ver el CI más allá de la aplicación del principio de autonomía. Para expertos en bioética, como Doucet Hubert (85), el tema amerita un abordaje especial, debido al cambio que se viene dando en la bioética con respecto a la búsqueda de las formas más apropiadas de incorporar mecanismos de análisis $e$ investigación, que aporten a la construcción de la reflexión en los diversos campos en los que está involucrada.

Aunque siguen siendo escasas, en la actualidad se escuchan voces que hablan de la necesidad de mirar a fondo los contextos socioculturales, pues la revisión de principios aislados o metodologías estandarizadas y cuantitativas en aspectos que tienen que ver con elementos cualitativos de las relaciones humanas, como los presentes en el CI resultan insuficientes. Con esta revisión se establece 
que una mayor proporción de escritos e investigaciones emplea metodologías y análisis cuantitativos para revisar aspectos humanos como la percepción del $\mathrm{CI}$ donde la influencia del contexto cultural, la condición socioeconómica o la biografía, inciden de manera importante y deberían impedir hacer generalizaciones o sistematizaciones estadísticas, las cuales resultan impropias. Si bien tener en cuenta la investigación de percepción es fundamental para cualificar la relación entre pacientes y profesionales de salud, es indispensable repensar las formas en que se hacen los abordajes y la interpretación que se hace de ellos, así como la priorización que se hace de los estudios a nivel de profesionales en comparación con los pocos estudios en pacientes.

Es igualmente problemático que, mediante el sondeo en grupos pequeños y localizados de población, en algunos estudios se realizan inferencias incluso para países enteros o para proceso de $\mathrm{CI}$ en general, pues se requieren estudios adicionales para hacer válida la generalización, sin los cuales no tiene fundamento. Si bien es importante seguir realizando esfuerzos en la comprensión de los complejos problemas alrededor del CI, y una forma muy valiosa son los estudios de caso, el análisis en circunstancias específicas no debe dar pie a retornar al boom de estudios cualitativos de poco rigor de hace pocas décadas que generó no solo gran malestar frente a esta valiosa herramienta investigativa, sino pérdida de credibilidad, debido a lo incierto de las generalizaciones. ${ }^{3}$

Algunos estudios tanto en investigación como en clínica describen problemas en su implementación, en cuanto a cantidad y calidad de la información, el establecimiento de la capacidad y competencia del sujeto o el ejercicio claro de la voluntad a la luz de parámetros culturales y sociales, por ejemplo el paternalismo y cabría agregar que en nuestros contextos debería abordarse el papel que juegan en las decisiones otros aspectos culturales como el machismo, las circunstancias socioeconómicas, el acceso a los servicios, etc.

$\mathrm{El} \mathrm{CI}$ es un proceso reciente que hoy goza no sólo de reconocimiento legal en salud, sino que también se ha convertido en una herramienta para la participación en la toma de decisiones de los pacientes. Si bien es cierto que, en general, en los escritos revisados se advierten

3 Un buen análisis al respecto, especialmente con relación a la validez y generalización de los estudios cualitativos, es Le Raisonnement Sociologique (1991), de es el realizado por Jean Claude Passeron. problemas en implementación y aplicación y que falta mejorar los abordajes teóricos e investigativos, cabe resaltar la preocupación creciente demostrada en los cada vez más numerosos estudios sobre el tema, los cuales se vuelven significativos para la cualificación del proceso con la participación de los pacientes.

\section{Agradecimientos}

Este artículo de revisión hace parte de la primera fase de la investigación HUM 1238 Percepción sobre consentimiento informado en diferentes áreas de atención en salud en la ciudad de Bogotá. Pertenece al grupo de investigación de la Facultad de Educación y Humanidades, Bioethics Group y es financiado por la vicerrectoría de investigaciones de la Universidad Militar Nueva Granada. Cuenta con el apoyo y revisión de la asistente profesional en investigación, Angie Arenas Piedrahita, politóloga; el asistente profesional en investigación, Cesar Carrera Celis, trabajador social; y la joven investigadora, Sandra Lorena Vargas, bióloga; quienes participaron en la búsqueda y sistematización de la información.

\section{Referencias}

1. Simon P. Consentimiento informado. Historia teoría y práctica. Madrid (España): Editorial Triacastela; 2000.

2. Escobar M. Consentimiento informado en odontología. Más que un problema legal, un asunto para reflexionar sobre la relación odontólogo-paciente y la odontología en general. Revista de la Federación Odontológica Colombiana. 2004; 21: 43-52.

3. Vargas L. Sobre el concepto de percepción. Alteridades [Serie en Internet]. 1994 [Citado 24 de Nov. 2014] pp. 47-53 Disponible en: http://www.scielo.org.mx/scielo. php? script $=$ sci_nlinks\&ref $=3036638 \&$ pid $=S 0188$ 2503201400010000300052\&lng=es

4. Benitez O, Dominique D, Jean D. Audiovisual documentation of oral consent a new method of informed consent for illiterate populations. The Lancet. 2002; 359(9315): 1406-7.

5. Lanckton A van C. The contours of informed consent. Survey of Ophtalmology. 1996; 40(5): 391-394 Cañete R, Guilhem D, Brito K. Consentimiento informado algunas consideraciones actuales. Acta Bioethica. 2012; 18(1): 121-7.

6. Cheatle M, Savage S. Informed Consent in Opioid Therapy. A Potential Obligation and Opportunity. Journal of Pain and Symptom Management. 2012; 44(1): 105-16.

7. Clarke S. Informed consent without bureaucracy. Journal of Clinical Neuroscience. 2003; 10(1): 35-6.

8. Delany C. Respecting patient autonomy and obtaining their informed consent ethical theory-missing in action. Physiotherapy. 2005; 91: 197-203. 
9. Gómez M, González F. Mejora de la calidad en el consentimiento informado. Acta Bioethica. 2012; 18(2): 247-56.

10. Gopal A, Cosgrove L, Shuv-Ami I, et al. Dynamic informed consent processes vital for treatment with antidepressants. International Journal of Law and Psychiatry. 2012; 35(56): 392-7.

11. Lynoe et. al. Obtaining informed consent in Banglades. The new England journal of medicine. 2001; 344: 460-1.

12. Mitchell E. Are women who are taking Hormone Replacement Therapy doing so with informed consent? Radiography. 2003; 9(4): 269-75.

13. Padhy B, Gupta P, Gupta YK, et al. Analysis of the compliance of informed consent documents with good clinical practice guideline. Contemporary Clinical Trials. 2011; 32(5): 662-6.

14. Piera y Ortega. Adaptación del consentimiento informado a la legislación española en los ensayos clínicos internacionales. Panace. 2012. 36; pp. 291-3.

15. Skillern A, Cedars M, Huddleston H, et al. Egg Donor Informed Consent Tool (EDICT) development and validation of a new informed consent tool for oocyte donors. Fertility and Sterility. 2013; 99(6): 1733-8.

16. Dávila J, Pérez R. Consentimiento informado de Medicina Interna en el Hospital Universitario San Ignacio. Universitas Médica. 2005; 46(3): 74-80.

17. Siqueira J. Los orígenes del consentimiento informado en clínica. Rev BioetLatinoam [Internet]. 2009 [citado 2016 Oct 14]; 3(3). Disponible en: http://www.saber.ula.ve/bitstream/123456789/27949/6.

18. Berrocal A. El consentimiento Informado y capacidad del paciente para prestarlo válidamente en la Nueva Ley 41/2002. Anuario de Derechos Humanos. 2004; 5: 11123.

19. Cabrales R, Calvachi J, Foronda J, et al. ¿Quién se informa con el consentimiento informado? Revista de Salud Pública. 2012 ; 14(3): 502-11.

29. Macías A. El Consentimiento Informado en Pediatría. Revista Cubana de Pediatría. 2006; 78(1): 78-84.

21. Sankar P. Communication and Miscommunication in Informed Consent of Research. Medical Anthropology Quarterly. 2004; 18(4): 429-46.

22. Kapp M. Patient Autonomy in the age of consumer-driven health care: informed consent and informed choice. Journal of Legal Medicine. 2007; 28(1): 91-177.

23. Reyes M, Águila Y, Llanes H, Robaina M, De Sanfiel M. Consentimiento Informado en pacientes sometidos a cirugía por mínimo acceso. Revista Cubana de Enfermería. 2007; 22 (4): 20-35.

24. Aguilar L. Consentimiento Informado en la Paciente embarazada menor de edad. Revista Mexicana de Anestesiología. 2008; 31(1): 238-42.

25. Figueroa R. Consentimiento Informado en la nueva Ley de derechos de los pacientes. Revista Médica de Chile. 2012; 140(10): 1347-51.
26. Siegal G, Bonnie R, Appelbaum P. Personalized Disclosure by Information -on-Demand: Attending to Patients' Needs in the Informed Consent Process. Journal of Law, Medicine \& Ethics. 2012; 40(2): 359-67.

27. Stoljar Natalie. Informed Consent and relational Conceptions of autonomy. Oxford University press Journal Of medice and philosophy. 2011; 36(4): 375-84.

28. García F. El odontólogo frente al consentimiento informado ¿Qué hacer? En: Cardozo C, Rodríguez E, Lolas F, Quezada A, et al. Ética y odontología una introducción. Chile: Centro Interdisciplinario de Estudios de Bioética; 2007; p. 105-15.

29. Wiesner C, Monsalve L. Interactuar, Comunicar, Deliberar. Un estudio de caso: el consentimiento informado en el Instituto Nacional de Cancerología. Bogotá: Universidad Externado de Colombia; 2009.

30. Galán JC. Responsabilidad Médica y Consentimiento Informado. Madrid: Civitas; 2001.

31. Castaño M. El Consentimiento Informado del Paciente en la Responsabilidad Médica. Bogotá: Temis; 1997.

32. Fajardo F, Ruano L. El Consentimiento Informado. Del paternalismo a la reivindicación de los derechos humanos del paciente. Bogotá: Ibáñez; 2009.

33. Gómez I, Soto C. La responsabilidad civil médica frente al Consentimiento Informado del Paciente. [Tesis]. Medellín: Universidad de Medellín; 2002.

34. Echavarría M, Martínez E. El Consentimiento Informado como prueba de responsabilidad médica. [Tesis]. Bogotá: Universidad Nacional de Colombia; 2002.

35. Luque M, Villota M. Consentimiento Informado en el paciente con enfermedad mental y su familia. [Tesis]. Bogotá; 1998.

36. Parra M. Una mirada bioética del proceso de consentimiento informado y su aplicación en el Instituto Materno Infantil "Concepción Villaveces de Costa". [Tesis]. Bogotá: Universidad el Bosque; 2002.

37. De Brigard A. Consentimiento Informado del Paciente. Revista Colombiana de Gastroenterología. 2004; 19(4): 277-80.

38. Devadiga A. Informed Consent and the Dentist. Online Journal of Health Ethics. 2012; 8(1): 1-20.

39. Herazo B. Consentimiento informado: Para procedimientos, intervenciones y tratamientos en Salud. Bogotá: Ecoe; 2007.

40. Lanckton A, Van C. The contours of informed consent. Survey of Ophtalmology. 1996; 40(5): 391-4.

41. Bismark M, Gogos A, McCombe D, et al. Legal disputes over informed consent for cosmetic procedures: A descriptive study of negligence claims and complaints in Australia. Journal of Plastic, Reconstructive \& Aesthetic Surgery. 2012; 65: 1506-12.

42. Hipshman L. Attitudes towards informed consent, confidentiality, and substitute treatment decisions in southern African medical students a case study from Zimbabwe. Social Science \& Medicine. 1999; 49(3): 313-28. 
43. Ovalle C. Práctica y significado del consentimiento informado en hospitales de Colombia y Chile. Estudio de casos. [Tesis doctoral]. Bogotá: Universidad el Bosque; 2009.

44. Oppliger W, Bascuñan M. Consentimiento informado. Percepción de médicos, enfermeras y padres sobre el proceso comunicativo. Revista Chilena de Pediatría. 2011; 82(3): 204-10.

45. Charry M, López J. Percepción sobre consentimiento informado en profesionales de los hospitales de Arauca y Fusagasugá. Bogotá: Universidad el Bosque; 2005.

46.Stoljar N. Informed Consent and Relational Conceptions of Autonomy. Journal of Medicine and Philosophy. 2011; 36(4): 375-84.

47. Brown R, Butow P, Ellis P, Boyle F, Tattersall M. Seeking informed consent to cancer clinical trials describing current practice. Social Science\& Medicine. 2004; 58 (12): 2445-57.

48. Cañete et al. Consentimiento informado algunas consideraciones actuals. Actha Bioethica 18; paginas 121-7.

49. Dawson L, Kass N. Views of US researchers about informed consent in international collaborative research. Social Science \& Medicine. 2005; 61 (6): 1211-22.

50. Ellenberg S. Informed consent Protection or obstacle? Some emerging issues. Controlled Clinical Trials. 1997; 18 (6): 628-36.

51. Eyler L, Mirzakhanian H, Jeste D. A preliminary study of interactive questioning methods to assess and improve understanding of informed consent among patients with schizophrenia. Schizophrenia Research. 2005; 75 (2-3): 193-8.

52. Gikonyo C, Bejon P, Marsh V, Molyneux S. Taking social relationships seriously Lessons learned from the informed consent practices of a vaccine trial on the Kenyan Coast. Social Science \& Medicine. 2008; 67 (5): 708-20.

53. Joffe S, Cook E, Cleary P, Clark J, Weeks J. Quality of informed consent in cancer clinical trials a cross-sectional survey. The Lancet. 2001; 358 (9295): 1772-7.

54. Pullman D, Wang X. Adaptive Designs, Informed Consent, and the Ethics of Research. Controlled Clinical Trials. 2001; 22 (3): 203-10.

55. Ordovás J. Estudio observacional y prospectivo del consentimiento informado de los pacientes en ensayos clínicos con medicamentos. Medicina Clínica. 2008; 131(11): 422-5.

56. Valle-Mansilla I, Ruiz-Canela M, Sulmasy D. Patients' Attitudes to Informed Consent for Genomic Research with Donated Samples. Cancer Investigation. 2010; 28 (7): 726-34.

57. Ayuningtyas D, Sondani V. Informed Consent Communication of Elective Surgery Patients in KaryaMedika I Hospital (RSKM I) Bekasi. Journal of US-China Medical Science. 2011 ; 8(6): 366-71.

58. Meneguin S, Zobolill E, Domingues R, et al. La comprensión del formulario de Consentimiento por los pacientes que forman parte de investigaciones con fármaco en la Cardiología. Arquivos Brasileiros de Cardiologia. 2010; 94(1): 4-9.
59. Garzón F. Aspectos bioéticos del consentimiento del consentimiento informado en investigación biomédica con población vulnerable. Bogotá: UMNG; 2010.

60. Fernández M, Ardura M. Adaptación del consentimiento informado a la legislación española en los ensayos clínicos internacionales. Panace. 2012; 13(36): 291-3.

61. Geller G, Tambor ES, Bernhardt BA, Fraser G, Wissow LS. Informed consent for enrolling minors in genetic susceptibility research a qualitative study of at-risk children's and parents' views about children's role in decision-making. Journal of Adolescent Health. 2003; 32: 260-71.

62. Clark S, Mangram A, Ernest D, et al. The Informed Consent. A Study of the Efficacy of Informed Consents and the Associated Role of Language Barriers. Journal of Surgical Education. 2011; 68(2): 143-7.

63. Enama M, Hu Z, Gordon I, et al. Randomization to standard and concise informed consent forms Development of evidence-based consent practices. Contemporary Clinical Trials. 2012; 33 (5): 895-902.

64. Fitzgerald D, Marotte C, Verdier R, et al. Comprehension during informed consent in a less-developed country. The Lancet. 2002; 360 (9342); 1301-2.

65. Hsiao-Lin H, Lian-Hua H, Fon-Jou H, Song-Nan C. Informed Consent for Antenatal Serum Screening for Down Syndrome. Taiwanese Journal of Obstetrics and Gynecology. 2010; 49(1): 50-6.

66. Kajja I, Bimenya G, Smit C. Informed consent in blood transfusion Knowledge and administrative issues in Uganda hospitals. Transfusion and Apheresis Science. 2011; 44: 33-9.

67. Guix J, Balañà L, Carbonell J, Simón R, et al. Cumplimiento y percepción del consentimiento informado en un sector sanitario de Cataluña. Revista Española de Salud Pública. 1999; 73: 669-75.

68. Fenety A, Harmana K, Hoensb A, Bassettd R. Informed consent practices of physiotherapists in the treatment of low back pain. Manual Therapy. 2009; 14 (6): 654-60.

69. Gori S, Greco M, Catania C, et al. A new informed consent form model for cancer patients. Preliminary results of a prospective study by the Italian Association of Medical Oncology (AIOM). Patient Education and Counseling. 2012; 87 (2): 243-9.

70. Hazen R, Drotar D, Kodish E. The role of the consent document in informed consent for pediatric leukemia trials. Contemporary Clinical Trials. 2007; 28(4): 401-8.

71. Kušec S, Oreskovic S, Skegro M, et al. Improving comprehension of informed consent. Patient Education and Counseling. 2006; 60(2): 294-300.

72. Leeper-Majors K, Veale J, Westbrook T, Reed K. The effect of standardized patient feedback in teaching surgical residents informed consent results of a pilot study. Current Surgery. 2003; 60(6): 615-22.

73. Gasull M, Cabrera E, Leino-Kilpi H, Välimäki M, Leomonidou $\mathrm{C}$, et al. Autonomía y consentimiento informado en centros sociosanitarios en Cataluña. Percepciones de ancianos y enfermeras en España (III). Enfermería Clínica. 2002; 12(1): 6-12. 
74. Nortes V, Serra M, Tella, M. El Consentimiento Informado en la Atención Primaria. Opinan los profesionales. Revista Calidad Asistencial. 2001; 16: 253-258.

75. Bello, K. Percepción del consentimiento informado en la unidad de cuidado intensivo pediátrico de un hospital universitario de Bogotá. Revista Colombiana de Bioética. 2011; 6(2): 8-25.

76. Jara S, Bravo N. Conocimiento sobre consentimiento informado en docentes de una clínica odontológica universitaria. Bogotá. Tesis; 2005.

77. Hung et. Al. An empirical study of the effectiveness of multimedia disclosure of informed consent A technology mediated learning perspective - 2011 48; paginas 135-44.

78. Shin-Yuan H, Kuo-Liang H, Wen-Ju Y. An empirical study of the effectiveness of multimedia disclosure of informed consent a technology mediated learning perspective. Information \& Management. 2011; 48: 135-44.

79. Négrier S, Lanier-Demma F, Lacroix-Kante V, et al. Evaluation of the informed consent procedure in cancer patients candidate to immunotherapy. European Journal of Cancer. $1995 ; 31(10)$ : 1650-2.
80. Nwomeh B, Hayes J, Caniano D, Upperman JS, Kelleher KJ. A Parental Educational Intervention to Facilitate Informed Consent for Emergency Operations in Children. Journal of Surgical Research. 2009; 152 (2): 258-63.

81. Olver I, Whitford H, Denson L, Peterson M, Olver S. Improving informed consent to chemotherapy. A randomized controlled trial of written information versus an interactive multimedia CD-ROM. Patient Education and Counseling. 2009; 74 (2): 197-204.

82. Bespali Y, De Pena M, Consens M. Derechos de los pacientes en Uruguay: percepción de los usuarios de hospitales. Revista Médica de Uruguay. 1995; 11: 81-98.

83 Cook S, Damato B, Marshall E, Salmon P. Reconciling the principle of patient autonomy with the practice of informed consent: decision-making about prognostication in uveal melanoma. Health Expect. 2011; 14(4): 383-96.

84. Hall DE, Div M, Morrison P, Nikolajski C, Fine M, Arnold $\mathrm{R}$, Zickmund SL. Informed consent for inguinal herniorrhaphy and cholecystectomy describing how patients make decisions to have surgery. The American Journal of Surgery. 2007; 204: 619-625.

85. Doucet H. Les méthodes empiriques, une nouveauté en bioethique? Revista Colombiana de Bioética. 2008; 3(2): 9-32. 